\title{
VITRECTOMÍA PARS PLANA, FACOEMULSIFICACIÓN E IMPLANTE DE LENTE INTRAOCULAR PARA EL MANEJO DE CATARATA Y RETINOPATÍA DIABÉTICA PROLIFERATIVA: COMPARACIÓN DE TÉCNICA QUIRÚRGICA COMBINADA VERSUS EN DOS TIEMPOS
}

\author{
PARS PLANA VITRECTOMY, PHACOEMULSIFICATION \\ AND INTRAOCULAR LENS IMPLANTATION FOR \\ THE MANAGEMENT OF CATARACT AND PROLIFERATIVE \\ DIABETIC RETINOPATHY: COMPARISON OF A COMBINED \\ VERSUS TWO-STEP SURGICAL APPROACH
}

\author{
RIVAS-AGUIÑO P ${ }^{1}$, GARCÍA-AMARIS RA², BERROCAL MH³ ${ }^{3}$ SÁNCHEZ JG², \\ RIVAS A ${ }^{2}$, ARÉVALO JF ${ }^{2}$
}

\section{RESUMEN}

Propósito: Describir las complicaciones intra- y postoperatorias y los resultados visuales en vitrectomía vía pars plana (VPP), facoemulsificación e implante de lente intraocular (LIO) en pacientes con catarata y retinopatía diabética proliferativa (RDP). Se realizo una comparación de la técnica combinada versus en dos tiempos quirúrgicos.

Método: Ensayo clínico intervencional retrospectivo no controlado. Se incluyeron 48 ojos de 48 pacientes consecutivos con RDP. Se analizaron 28 $(58,3 \%)$ ojos con cirugía combinada y 20 ojos $(41,7 \%)$ con cirugía secuencial.

Resultados: El tiempo de seguimiento postoperatorio fue entre 6 y 63 meses (promedio: 18 meses). 1) Cirugía combinada: Agudeza visual mejor corregida preoperatoria (AVMC) estaba entre 20/200 a movimiento de manos, y AVMC postoperatoria se encon-

\section{ABSTRACT}

Purpose: To report the intra-and postoperative complications and visual acuity outcomes in pars plana vitrectomy (PPV), phacoemulsification and intraocular lens (IOL) implantation in patients with cataract and proliferative diabetic retinopathy (PDR). A comparison of the combined versus twostep surgical approach is given.

Method: Retrospective uncontrolled interventional clinical trial. Forty-eight eyes of 48 consecutive patients with PDR were included. Twenty-eight (58.3\%) eyes with combined surgery and 20 (41.7\%) eyes with sequential surgery were analyzed. Results: Postoperative follow-up time was between 6 and 63 months (mean: 18 months). 1) Combined surgery: Preoperative best-corrected visual acuity (BCVA) ranged from 20/200 to hand motions, and postoperative BCVA ranged from 20/30 to hand

\footnotetext{
Recibido: 23/3/07. Aceptado: 5/1/09.

Servicio de Retina y Vítreo, Clínica Oftalmológica Centro Caracas, Caracas, Venezuela.

${ }^{1}$ Doctor en Medicina. Departamento de Oftalmología, Universidad de Los Andes, Mérida, Venezuela.

2 Doctor en Medicina. Servicio de Retina y Vítreo, Clínica Oftalmológica Centro Caracas, Caracas, Venezuela.

${ }^{3}$ Doctor en Medicina. Universidad de Puerto Rico, San Juan, Puerto Rico.

Correspondencia:

J. Fernando Arévalo

Clínica Oftalmológica Centro Caracas

Edif. Centro Caracas PH-1

Av. Panteón, San Bernardino, Caracas 1010

Venezuela

E-mail: arevalojf2020@gmail.com
} 
tró entre 20/30 a movimiento de manos. En diecisiete $(60,7 \%)$ ojos la AVMC mejoró, en 7 (25\%) ojos no hubo cambios ( $\geq 2$ líneas ETDRS) en la AV y en $4(14,3 \%)$ ojos la AVMC se redujo. Las complicaciones postoperatorias incluyeron hemorragia vítrea (HV) en 10 ojos (35,7\%), y exudación fibrinosa en nueve ojos $(32,1 \%)$. 2) Cirugía en dos tiempos: AVMC preoperatoria entre 10/200 a percepción de luz, y de 20/40 a percepción de luz en el período postoperatorio. La agudeza visual mejor corregida mejoró en 15 (75\%) ojos, permaneció igual en cuatro (20\%) ojos, y se redujo en un (5\%) ojo. Las complicaciones postoperatorias incluyeron exudación fibrinosa en seis (30\%) ojos, y HV en tres (15\%) ojos.

Conclusión: Tanto la cirugía combinada de VPP, facoemulsificación e implante de LIO como el procedimiento en dos tiempos son seguras y efectivas para el manejo de catarata en RDP. La cirugía secuencial podría ser ventajosa para los resultados de AVMC al disminuir la posibilidad de HV postoperatoria, que parece ser más frecuente después de la cirugía combinada.

Palabras clave: Cirugía combinada, cirugía en dos tiempos, facoemulsificación, implante de lente intraocular, retinopatía diabética proliferativa, vitrectomía vía pars plana. motions. BCVA improved in 17 eyes $(60.7 \%)$, while in $7(25 \%)$ eyes there was no change ( $\geq 2$ ETDRS lines) in VA, and in $4(14.3 \%)$ eyes BCVA decreased. Postoperative complications included vitreous hemorrhage (VH) in $10(35.7 \%)$ eyes, and fibrinous exudation in 9 (32.1\%) eyes. 2) Two-step surgery: Preoperative BCVA ranged from 10/200 to light perception, and from 20/40 to light perception in the postoperative period. Best-corrected visual acuity improved in $15(75 \%)$ eyes, remained the same in $4(20 \%)$ eyes, and decreased in $1(5 \%)$ eye. Postoperative complications included fibrinous exudation in $6(30 \%)$ eyes, and $\mathrm{VH}$ in $3(15 \%)$ eyes. Conclusion: Combined PPV, phacoemulsification and IOL implantation as well as the two-step procedure are safe and effective for the management of cataract in PDR. Sequential surgery could be advantageous to BCVA outcomes by minimizing postoperative $\mathrm{VH}$, which is significantly more frequent after combined surgery (Arch Soc Esp Oftalmol 2009; 84: 31-38).

Key words: Combined surgical approach, intraocular lens implantation, pars plana vitrectomy, phacoemulsification, proliferative diabetic retinopathy, two-step surgical approach.

\section{INTRODUCCIÓN}

Las enfermedades vítreo-retinales y las cataratas frecuentemente ocurren de manera simultanea en pacientes con retinopatía diabética proliferativa (RDP). Los progresos en las técnicas quirúrgicas para la extracción de catarata y la mejoría en la tecnología de las lentes intraoculares (LIO) han incrementado las indicaciones para la cirugía de catarata en los pacientes diabéticos. Además, la vitrectomía vía pars plana (VPP) es realizada ahora con éxito en diversas enfermedades vítreo-retinales. La extracción de catarata puede ser combinada con VPP si las opacidades del cristalino interfieren con la visualización adecuada de la retina dificultando la cirugía. Aún, si la catarata no es significativa al momento de la vitrectomía, esta puede progresar posteriormente, con un porcentaje de entre $68 \%$ y $80 \%$ a dos años de seguimiento, siendo mucho mas rápida en los pacientes diabéticos (1-4). Sin embargo, otros factores predisponentes para la formación de catarata incluyen la edad del paciente, la esclerosis nuclear pre-existente, daño del cristalino durante VPP y el uso de aceite de silicona y gas intravítreo (5-7). Los métodos para la extracción de catarata incluyen lensectomía, extracción extracapsular y facoemulsificación. La facoemulsificación tiene ciertas ventajas sobre las otros procedimientos quirúrgicos debido a la rápida recuperación visual y a la menor inflamación postoperatoria $(8,9)$.

Los procedimientos para remover cataratas y reparar enfermedades del segmento posterior pueden ser realizadas de manera secuencial con un procedimiento en dos tiempos quirúrgicos: cirugía del segmento posterior y luego extracción del cristalino; o en una sesión combinada de cirugía de catarata y de patología vítreo-retinal en un solo tiempo (10). La cirugía de catarata en ojos vitrectomizados representa circunstancias especiales que incluyen perdida del soporte vítreo, inestabilidad de la capsula posterior, debilidad de las zónulas y placas capsulares posteriores $(11,12)$. Existen pocos traba- 
jos que comparen directamente los resultados de las cirugías combinadas con los obtenidos mediante procedimientos secuenciales; sin embargo, estos resultados son controvertidos (3,13-18).

En este estudio analizamos retrospectivamente las complicaciones intra- y postoperatorias, las dificultades y los resultados de combinar VPP, facoemulsificación e implante de LIO en pacientes con catarata en RDP, y estos fueron comparados con los obtenidos en pacientes que fueron sometidos a facoemulsificación e implante de LIO como un procedimiento secundario después de vitrectomía.

\section{SUJETOS, MATERIAL Y MÉTODOS}

Se revisaron retrospectivamente los expedientes médicos y quirúrgicos de 48 ojos de 48 pacientes consecutivos con RDP y catarata. A 28 (58,3\%) ojos se les realizo VPP combinada con facoemulsificación e implante de LIO (cirugía combinada) y a veinte $(41,7 \%)$ ojos se les realizo facoemulsificación e implante de LIO después de vitrectomía (cirugía secuencial). Todos los pacientes tenían una opacidad del cristalino clínicamente significativa a la hora de cirugía. La cirugía combinada fue realizada cuando la catarata era bastante significativa y deterioraba la visualización del polo posterior. La cirugía secuencial se realizo cuando la catarata no era lo bastante significativa para deteriorar la visualización del polo posterior. Los pacientes fueron operados en nuestros centros entre enero de 2002 y diciembre de 2005, previa aprobación del comité de ética institucional y la obtención de consentimiento informado. Además, este estudio fue realizado de acuerdo con los estándares éticos de la declaración de Helsinki de 1964.

Treinta y seis ojos (75\%) tenían fotocoagulación panretinal (PRP) previa. Los criterios de exclusión incluyeron isquemia macular determinada por angiografía fluoresceínica (cuando era posible). Todos los procedimientos fueron realizados bajo anestesia local peribulbar monitorizada. En el grupo de cirugía secuencial, la extracción de la catarata fue realizada en ojos previamente vitrectomizados cuando el estado retiniano era estable y la catarata subsecuente era considerada la causa de la pérdida visual. En los 48 casos, la técnica de la facoemulsificación fue similar, y en el grupo de procedimientos combinados la extracción de la catarata precedió a la cirugía vitreorretinal. Una incisión en córnea clara de 3,2 $\mathrm{mm}$ de ancho y 1,5-2,0 mm de longitud fue creada en el limbo temporal, la capsulorrexis curvilínea de 5,0 a 6,0 mm fue completada, y la facoemulsificación y la extracción de la corteza fueron realizadas. La cámara anterior y el saco capsular fueron llenados con viscoelástico y la LIO plegable fue implantada. El túnel corneal fue temporalmente cerrado con un punto de nylon 10-0. A todos los pacientes con cirugía combinada, una vitrectomía vía pars plana estándar de tres puertos con una fuente de luz intraocular, un instrumento de vitrectomía y una cánula de infusión suturada en el sitio de la esclerotomía inferotemporal fue usada para la cirugía vitreorretinal. Las esclerotomías fueron realizadas a 3,5 (fáquicos) o 2,5 (pseudofáquicos) $\mathrm{mm}$ posteriores al limbo en los cuadrantes superotemporales, superonasales o inferotemporales. Las esclerotomías y la conjuntiva fueron suturadas. Los desgarros retinales fueron tratados inmediatamente en caso de presentarse. La cirugía combinada y la cirugía secuencial fueron realizadas por tres cirujanos (PRA, MHB, y JFA).

La información siguiente fue obtenida para cada paciente: edad, sexo, agudeza visual mejor corregida (AVMC) pre y postoperatoria, presión intraocular (PIO), indicación para la cirugía vitreorretinal, observaciones y complicaciones intraoperatorios, longitud del seguimiento, complicaciones postoperatorias (glaucoma neovascular, exudación fibrinosa, desprendimiento de retina, edema macular y hemorragia vítrea recurrente) y procedimientos quirúrgicos adicionales.

Un examen oftalmológico de rutina fue realizado preoperatoriamente y al primer día, a la semana y 1 , $3,6,12$ y 18 meses postoperatorios en todos los pacientes. Este examen incluyo AVMC, tonometría de aplanación, biomicroscopía y examen del fondo por oftalmoscopia directa e indirecta. La AVMC y sin corregir fue medida usando las cartilla del ETDRS. En nuestro estudio, se consideró la agudeza visual como estable cuando la variación fue menor o igual a dos líneas ETDRS. Los resultados visuales y las complicaciones fueron comparados entre los grupos.

\section{RESULTADOS}

En este estudio los pacientes tuvieron un seguimiento promedio de 18 meses (rango: 6 a 63 meses). La edad media de nuestros pacientes fue de 
Tabla I. Datos Demográficos de los Pacientes*

\begin{tabular}{|c|c|c|}
\hline & $\begin{array}{c}\text { Cirugía } \\
\text { Combinada }\end{array}$ & $\begin{array}{c}\text { Cirugía } \\
\text { Secuencial }\end{array}$ \\
\hline No. de ojos & $28(58,3 \%)$ & $20(41,7 \%)$ \\
\hline \multicolumn{3}{|l|}{ Edad (años) } \\
\hline Promedio & 57 & 59 \\
\hline Rango & $36-76$ & $28-82$ \\
\hline \multicolumn{3}{|l|}{ Sexo } \\
\hline Femenino & $17(60,7 \%)$ & $6(30 \%)$ \\
\hline Masculino & $11(39,3 \%)$ & $14(70 \%)$ \\
\hline \multicolumn{3}{|l|}{ Seguimiento (meses) } \\
\hline Promedio & 16.5 & 19,8 \\
\hline Rango & $6-63$ & $9-40$ \\
\hline \multicolumn{3}{|c|}{ Agudeza Visual (ETDRS) } \\
\hline Rango preoperatorio & 20/200 - MM & $10 / 200-\mathrm{PL}$ \\
\hline Rango postoperatorio & 20/30 - MM & 20/40 - PL \\
\hline \multicolumn{3}{|c|}{$\begin{array}{l}\text { * ETDRS = Early Treatment Diabetic Retinopathy Study, MM = } \\
\text { Movimiento de manos, PL = Percepción de luz. }\end{array}$} \\
\hline \multicolumn{3}{|c|}{ Tabla II. Indicaciones para Vitrectomía* } \\
\hline \multirow[b]{2}{*}{ Indicación } & \multicolumn{2}{|c|}{ Grupos de Estudio } \\
\hline & $\begin{array}{c}\text { Cirugía } \\
\text { Combinada } \\
(n=28)\end{array}$ & $\begin{array}{c}\text { Cirugía } \\
\text { Secuencial } \\
(n=20)\end{array}$ \\
\hline Hemorragia Vítrea & $17(60,7 \%)$ & $13(65 \%)$ \\
\hline DRT & $11(39,3 \%)$ & $7(35 \%)$ \\
\hline
\end{tabular}

* DRT $=$ Desprendimiento de Retina Traccional.

Tabla III. Resultados de Agudeza Visual*

\begin{tabular}{lcc}
\hline \multirow{2}{*}{ Agudeza Visual (ETDRS) } & \multicolumn{2}{c}{ Ojos (\%) } \\
\cline { 2 - 3 } & $\begin{array}{c}\text { Cirugía } \\
\text { Combinada } \\
(\mathrm{n}=28)\end{array}$ & $\begin{array}{c}\text { Cirugía } \\
\text { Secuencial } \\
(\mathrm{n}=20)\end{array}$ \\
\hline Mejoría $\geq 2$ líneas & $17(60,7 \%)$ & $15(75 \%)$ \\
Dentro de 2 líneas & $7(25 \%)$ & $4(20 \%)$ \\
Disminución $\geq 2$ líneas & $4(14,3 \%)$ & $1(5 \%)$ \\
\hline \hline
\end{tabular}

* ETDRS $=$ Early Treatment Diabetic Retinopathy Study.

59 (rango: 28-82) años en el grupo de cirugía secuencial y de 57 (rango: 36-76) años en el grupo de cirugía combinada. El setenta por ciento de pacientes en el grupo de cirugía secuencial eran hombres comparado con el 39,3\% en el grupo de cirugía combinada (tabla I). Las indicaciones primarias para vitrectomía en ambos grupos se resumen en la tabla II. Las indicaciones fueron la hemo-
Tabla IV. Complicaciones Postoperatorias

\begin{tabular}{lcc}
\hline \multirow{2}{*}{ Complicaciones } & \multicolumn{2}{c}{ Ojos (\%) } \\
\cline { 2 - 3 } & $\begin{array}{c}\text { Cirugía } \\
\text { Combinada } \\
(\mathrm{n}=28)\end{array}$ & $\begin{array}{c}\text { Cirugía } \\
\text { Secuencial } \\
(\mathrm{n}=20)\end{array}$ \\
\hline Exudación Fibrinosa & $9(32,1 \%)$ & $6(30 \%)$ \\
Glaucoma Neovascular & $3(10,7 \%)$ & $3(15 \%)$ \\
Membrana Prepupilar & $3(10,7 \%)$ & $1(5 \%)$ \\
Edema Macular & $5(17,8 \%)$ & $4(20 \%)$ \\
Hemorragia Vítrea & $10(35,7 \%)$ & $3(15 \%)$ \\
Desprendimiento de Retina & - & $1(5 \%)$ \\
Opacificación Capsula Posterior & $1(3,5 \%)$ & $1(5 \%)$ \\
\hline \hline
\end{tabular}

rragia vítrea (HV) (cirugía combinada: 60,7\%, cirugía secuencial: $65 \%$ ) y el desprendimiento de retina traccional (DRT) (cirugía combinada: 39,3\%, cirugía secuencial: $35 \%$ ).

La endofotocoagulación láser fue utilizada en todos los pacientes de ambos grupos. El endoláser y la criopexia combinados fueron necesarios solamente en un caso del grupo de cirugía combinada. Para el endotaponamiento después de vitrectomía, el agente más frecuentemente usado fue el gas $\mathrm{C} 3 \mathrm{~F} 8$ (cirugía combinada: $42,8 \%$, cirugía secuencial: $30 \%$ ). El aceite de silicona fue utilizado en un $(3,5 \%)$ ojo en el grupo de cirugía combinada y en dos ojos (10\%) del grupo de cirugía secuencial. El aire fue utilizado en seis $(21,4 \%)$ casos en el grupo de cirugía combinada. En el grupo de cirugía secuencial la cirugía de catarata se realizo en un promedio de 4,5 meses (rango: 2-9 meses) posterior a la VPP.

En el grupo de cirugía combinada, la AVMC preoperatoria estaba entre 20/200 y movimiento de manos (promedio: 5/200) y la AVMC postoperatoria entre 20/30 y movimiento de manos (promedio: 10/200). En diecisiete (60,7\%) ojos la AVMC mejoró, en siete ojos $(25 \%)$ no hubo cambios ( $\geq 2$ líneas ETDRS) en la AVMC, y en cuatro (14,3\%) ojos la AVMC disminuyó. En comparación, la AVMC preoperatoria del grupo de cirugía secuencial oscilaba entre 10/200 y percepción de luz (promedio: 5/200) y entre 20/40 y percepción de luz (promedio: 10/200) en el período postoperatorio. La agudeza visual mejor corregida mejoró en quince ojos (75\%), permaneció igual en cuatro ojos $(20 \%)$ y disminuyó en un ojo (5\%) (tabla III).

Las complicaciones fueron observadas en 18 ojos $(64,3 \%)$ en el grupo de cirugía combinada y en once ojos $(55 \%)$ en la secuencial. Las complicaciones intraoperatorias incluyeron desgarros retinianos 
(combinada: $7,1 \%$, secuencial: $15 \%$ ), hemorragia subretinal (combinada: 3,5\%) y desgarros capsulares posteriores (combinada: 3,5\%, secuencial: 5\%). La tabla IV describe las complicaciones postoperatorias de este estudio. Las complicaciones mas comunes del grupo combinado fueron hemorragia vítrea en diez $(35,7 \%)$ ojos y exudación fibrinosa en nueve $(32,1 \%)$; mientras que la HV fue observada en tres ojos $(15 \%)$ y exudación fibrinosa en seis ojos $(30 \%)$ en el grupo secuencial. La formación de una membrana prepupilar fue más frecuente después de cirugía combinada $(10,7 \%)$ que en el grupo de cirugía secuencial (5\%). El glaucoma neovascular (GNV) ocurrió en tres $(10,7 \%)$ ojos de la cirugía combinada y en tres ojos (15\%) vitrectomizados previamente. Sin embargo, este fue controlado médicamente con medicamentos anti-glaucomatosos tópicos, excepto en un paciente del grupo de cirugía combinada que requirió cirugía filtrante. En el grupo de cirugía combinada, se evidencio edema macular en cinco $(17,8 \%)$ ojos. El grupo de cirugía secuencial se observo edema macular en cuatro (20\%) ojos. Complicaciones adicionales incluyeron opacificación de la cápsula posterior en un $(3,5 \%)$ ojo del grupo combinado y en un ojo (5\%) del grupo de cirugía secuencial. Solamente un caso de desprendimiento de retina postoperatorio se presento en este estudio y fue en el grupo de cirugía secuencial. No hubo ningún caso de endoftalmitis, ni de descentramiento o captura de la LIO en ninguno de los grupos.

\section{DISCUSIÓN}

Una de las secuelas mas comunes de la vitrectomía es la formación y progresión de cataratas, que oscila entre el $17 \%$ y el $80 \%$ (1-6,17). Los pacientes diabéticos tienden a tener mas opacidades del cristalino preoperatoriamente, que otros pacientes de la misma edad y frecuentemente después de la vitrectomía experimentan de manera temprana opacidades cristalinianas $(3,8,15)$. El ojo diabético es también mas susceptible al trauma quirúrgico que el ojo no diabético. Una miosis pronunciada quirúrgicamente, una capsula del cristalino mas frágil con un incrementado porcentaje de ruptura, una mayor duración de la cirugía, una elevación transitoria de la PIO, una mayor intensidad de inflamación postoperatoria y una mas alta incidencia de edema macular cistoideo han sido reportados en ojos diabéticos (8).
En casos donde la catarata coexista con una patología vitreorretinal, los cirujanos luchan por optimizar la visualización de la retina durante la cirugía vítrea y mejorar la rehabilitación visual postoperatoria para sus pacientes minimizando las complicaciones. La cuestión de remover o no la catarata en el mismo tiempo quirúrgico con la VPP ha sido poco estudiada y la combinación es atractiva en un intento para acelerar la recuperación visual del paciente, previniendo así, la necesidad de una segunda cirugía. La cirugía combinada es preferida por algunos cirujanos, debido a que tiene algunas ventajas como un tiempo mas corto de recuperación postoperatoria, una extracción completa de las estructuras vítreas anteriores sin el riesgo de lesionar el cristalino, una buena visualización del polo posterior durante la vitrectomía y la necesidad de una sola operación, lo cual reduce las molestias del paciente y disminuye los riesgos y los costos (2,3,13-15). Además, a los pacientes diabéticos se les realiza frecuentemente fotocoagulación panretinal como parte de la cirugía, disminuyendo el riesgo de desarrollar neovascularización de la retina y del iris $(3,13,17)$. Sin embargo, existen posibles desventajas, tales como, incremento en el tiempo quirúrgico y en la exigencia del cirujano, dificultad en la visualización de la capsulorrexis debido a la ausencia o reducción del reflejo rojo, dehiscencia de la herida quirúrgica por manipulación del globo durante la cirugía vítrea, miosis intraoperatoria posterior a la extracción de la catarata, sangrado de estructuras anteriores, perdida de la transparencia corneal por edema y pliegues en la membrana de Descemet, intercambio inadvertido de fluidos del segmento anterior con agentes de taponamiento del segmento posterior, descentración de la LIO y captura del iris en ojos con taponamiento de gas, aire o aceite de silicona, y efectos prismáticos y reflejos lumínicos no deseados durante la cirugía vitreorretinal causados por la implantación de la LIO antes del procedimiento en el segmento posterior $(2,3$, $5,13,14)$.

Se han descrito dificultades y desafíos durante una cirugía secuencial. Cámaras anteriores extremadamente profundas durante la facoemulsificación, dehiscencia zonular, movilidad incrementada de la capsula posterior y perdida de fragmentos nucleares se han reportado debido a la disminución del soporte vítreo $(1,3,6,14)$. Además, la cirugía de catarata en pacientes a quienes previamente se les realizo una VPP implica un mayor tiempo de recu- 
peración, dos anestesias locales o generales y una biometría frecuentemente inexacta (6). La cirugía de catarata en pacientes diabéticos puede también llevar a un empeoramiento de la retinopatía diabética $(3,6,8,15)$.

Existen algunos trabajos de resultados de cirugía combinada en catarata y enfermedades retinales $(1,3,9,10,15-19)$. Esos trabajos demuestran que la extracción de catarata combinada con VPP es segura y efectiva con una rápida recuperación visual. Sin embargo, aunque unos pocos estudios han comparado directamente los resultados de cirugía combinada con los de cirugía secuencial, para nuestro conocimiento, ninguno ha sido hecho en pacientes diabéticos $(2,13,14)$.

En este estudio, durante el tiempo de seguimiento (promedio: 18 meses; rango: 6 a 63 meses) revisamos y comparamos los resultados de cirugía combinada y secuencial en pacientes con RDP. El porcentaje de indicaciones para vitrectomía fue similar en ambos grupos siendo los mas comunes la hemorragia vítrea y el desprendimiento de retina traccional. La AVMC en el grupo de cirugía combinada mostró mejoría en diecisiete $(60,7 \%)$ ojos, estabilidad en siete $(25 \%)$ ojos y empeoramiento en cuatro (14,3\%) ojos. En el grupo de cirugía secuencial encontramos que la agudeza visual mostró mejoría en quince $(75 \%)$ ojos, estabilidad en cuatro (20\%) ojos y empeoramiento en un $(5 \%)$ ojo. El análisis de la AVMC entre los grupo de estudio revelo una importante diferencia con mejores resultados en el grupo de cirugía secuencial. Sin embargo, nuestro estudio esta limitado por su naturaleza retrospectiva, el pequeño número de casos y su diseño no randomizado.

Nosotros encontramos diferencias no significativas en las complicaciones intra- y postoperatorias entre el grupo de cirugía combinada y secuencial. Sin embargo, un mayor porcentaje de hemorragia vítrea fue notado en el grupo de cirugía combinada $(35,7 \%$ vs $15 \%)$. Un porcentaje similar de reacción inflamatoria en cámara anterior (exudación fibrinosa y desarrollo de membranas prepupilares) fue observado en ambos grupos. Chung y col (2), y Treumer y col (14) describieron diferencias similares en los porcentajes de exudación fibrinosa entre el grupo de cirugía combinada y secuencial. El glaucoma neovascular también tuvo similar frecuencia en ambos grupos. Sin embargo, este fue controlado médicamente con medicamentos antiglaucomatosos tópicos; solo un paciente requirió cirugía filtrante en el grupo de cirugía secuencial. Esta complicación ha sido descrita en la literatura como una complicación asociada a los procedimientos combinados (16). Diferencias en las complicaciones postoperatorias adicionales tales como edema macular, opacificación y desgarro de la capsula posterior y desprendimiento de retina no fueron significativas.

En resumen, tanto la combinación de VPP, facoemulsificación e implante de LIO como el procedimiento secuencial son seguros y efectivos en RDP. La cirugía secuencial podría tener cierta ventaja en los resultados de AVMC al disminuir la posibilidad de hemorragia vítrea postoperatoria, la cual parece ser mas frecuente después de una cirugía combinada. Una futura evaluación con un mayor número de pacientes en un estudio prospectivo randomizado es necesaria para determinar exactamente cualquier diferencia en los resultados entre ambas técnicas.

\section{BIBLIOGRAFÍA}

1. Grusha YO, Masket S, Miller KM. Phacoemulsification and lens implantation after pars plana vitrectomy. Ophthalmology 1998; 105:287-294.

2. Chung TY, Chung H, Lee JH. Combined surgery and sequential surgery comprising phacoemulsification, pars plana vitrectomy, and intraocular lens implantation: comparision of clinical outcomes. J Cataract Refract Surg 2002; 28:2001-2005.

3. Lahey JM, Francis RR, Kearney JJ. Combining phacoemulsification with pars plana vitrectomy in patients with proliferative diabetic retinopathy: a series of 223 cases. Ophthalmology 2003;110:1335-1339.

4. Melberg NS, Thomas MA. Nuclear sclerotic cataract after vitrectomy in patients younger than 50 years of age. Ophthalmology 1995; 102:1466-1471.

5. Hsuan JD, Brown NA, Bron AJ, Patel CK, Rosen PH. Posterior subcapsular and nuclear cataract after vitrectomy. J Cataract Refract Surg 2001;27:437-444.

6. Biro Z, Kovacs B. Results of cataract surgery in previously vitrectomized eyes. J Cataract Refract Surg 2002; 28:1003-1006.

7. Blodi BA, Paluska SA. Cataract after vitrectomy in young patients. Ophthalmology 1997;104:1092-1095.

8. Zaczek A, Olivestedt G, Zetterstrom C. Visual outcome after phacoemulsification and IOL implantation in diabetic patients. Br J Ophthalmol 1999;83:1036-1041.

9. Yang CQ, Tong JP, Lou DH. Surgical results of pars plana vitrectomy combined with phacoemulsification. J Zhejiang Univ Sci B 2006; 7:129-132.

10. Pollack A, Landa G, Kleinman G, Katz H, Hauzer D, Bukelman A. Results of combined surgery by phacoemulsification and vitrectomy. Isr Med Assoc J 2004;6:143146. 
11. Ahfat FG, Yuen CHW, Groenewald CP. Phacoemulsification and intraocular lens implantation following pars plana vitrectomy: a prospective study. Eye 2003;17:16-20.

12. Chang MA, Parides MK, Chang S, Braunstein RE. Outcome of phacoemulsification after pars plana vitrectomy. Ophthalmology 2002;109:948-954.

13. Senn P, Schipper I, Perren B. Combined pars plana vitrectomy, phacoemulsification, and intraocular lens implantation in the capsular bag: a comparison to vitrectomy and subsequent cataract surgery as a two-step procedure. Ophthalmic Surg Lasers 1995;26:420-428.

14. Treumer F, Bunse A, Rudolf M, Roider J. Pars plana vitrectomy, phacoemulsification and intraocular lens implantation. Comparison of clinical complications in a combined versus two-step surgical approach. Graefes Arch Clin Exp Ophthalmol 2006;244:808-815.

15. Belfort Mattos A, de Oliveira Bonomo PP, Freitas LL, Farah ME, Flynn H Jr, Bastos Pereira M. Phacoemulsification, pars plana vitrectomy and intraocular lens implant in eyes with proliferative diabetic retinopathy. Arq Bras Oftalmol 2004;67:441-449.

16. Demetriades AM, Gottsch JD, Thomsen R, Azab M, Stark WJ, Campochiaro PA, et al. Combined phacoemulsification, intraocular lens implantation, and vitrectomy for eyes with coexisting cataract and vitreorretinal pathology. Am J Ophthalmol 2003;135:291-296.

17. Hurley C, Barry P. Combined endocapsular phacoemulsification, pars plana vitrectomy, and intraocular lens implantation. J Cataract Refract Surg 1996;22:462-466.

18. Amino K, Tanihara H. Vitrectomy combined with phacoemulsification and intraocular lens implantation for diabetic macular edema. Jpn J Ophthalmol 2002;46:455-459.

19. Hsu $S Y, W u$ WC. Comparison of phacoemulsification and planned extracapsular cataract extraction in combined pars plana vitrectomy and posterior chamber intraocular lens implantation. Ophthalmic Surg Lasers Imaging 2005;36:108-113. 
